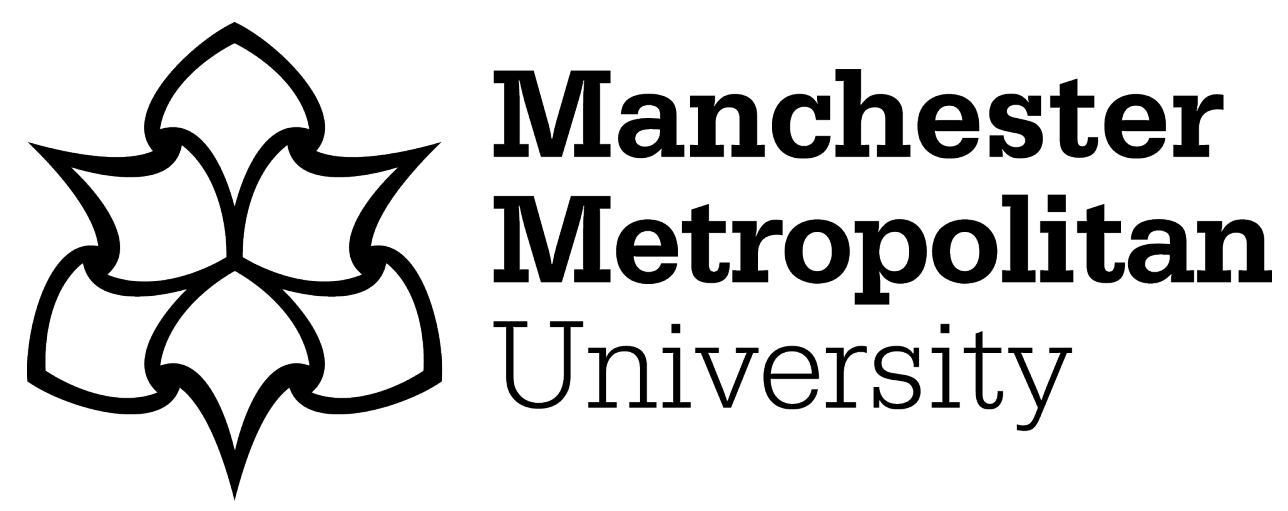

Preindl, Raphael, Nikolopoulos, Konstantinos and Litsiou, Konstantia (2020) Transformation Strategies for the Supply Chain: The Impact of Industry 4.0 and Digital Transformation. Supply Chain Forum: an International Journal, 21 (1). pp. 26-34. ISSN 1625-8312

Downloaded from: https://e-space.mmu.ac.uk/624685/

Version: Accepted Version

Publisher: Taylor \& Francis

DOI: https://doi.org/10.1080/16258312.2020.1716633

Please cite the published version 


\title{
Transformation Strategies for the Supply Chain: The Impact of Industry 4.0 and Digital Transformation
}

\section{Raphael Preindl ${ }^{a}$, Konstantinos Nikolopoulos ${ }^{\text {, }}$ *}

\author{
and Konstantia Litsiou ${ }^{\mathrm{c}}$ \\ ${ }^{a}$ Institute of Supply Chain Management, University of St.Gallen, St.Gallen, Switzerland; \\ ${ }^{b}$ forLAB, Bangor Business School, Bangor University, Bangor, United Kingdom \\ ${ }^{c}$ Manchester Metropolitan University Business School, Manchester, United Kingdom
}

Raphael Preindl

Research Associate

Institute of Supply Chain Management

University of St.Gallen (HSG) | Dufourstrasse 40a | CH-9000 St. Gallen

Tel. +41712247279

raphael.preindl@unisg.ch | iscm.unisg.ch

* Konstantinos Nikolopoulos (corresponding author)

Chair in Business Analytics, Bangor Business School

Bangor University, Bangor | Gwynedd LL57 2DG

Tel. +44 1248383796

kostas@bangor.ac.uk | bangor.ac.uk/business/

Konstantia Litsiou

Senior Lecturer in Retail Management, Department of Marketing, Retail and Tourism

Manchester Metropolitan University Business School

All Saints Campus, Oxford Road, Manchester, M15 6BH, UK.

k.litsiou@mmu.ac.uk |https://www2.mmu.ac.uk/business-school/ 


\title{
Transformation Strategies for the Supply Chain: The Impact of Industry 4.0 and Digital Transformation
}

\author{
This research focuses on the impact of 'Industry 4.0' and 'Digital Transformation' \\ on information sharing and decision making across the supply chain (SC). \\ Following a qualitative approach, the findings are threefold: First, it is shown that \\ the possibility of an entire SC integration based on new technologies is still at \\ distance. Current burdens are the missing willingness to exchange far-reaching \\ information even with long-term partners and the missing technological interface \\ standards in order to enable a trouble-free communication alongside the SC. \\ Second, the impact of Industry 4.0 and the Digital Transformation on decision \\ making is greatly connected to information sharing. An increasing amount of \\ decisions is prepared, recommended or even fully automated by information \\ systems. However, usually, the human being still has the last word. Third, \\ companies' preparations for these impacts differ greatly. Whereas some \\ companies rely on classical phase-based strategies and long-term visions, others \\ do not have a long-term plan at all.
}

Keywords: Supply Chain Management, Industry 4.0, Digital Transformation, Information Sharing, Decision Making

\section{Introduction}

The digitisation is one of the dominant topics in these times. Almost every area of life is affected by these innovations. Emerging technologies such as mobile devices, drones or self-driving cars, revolutionise everyday life of entire societies. This development also does not stop when it comes to companies and the way they are doing their daily business. The so-called 'Digital Transformation' and the connected area of 'Industry 4.0 ' are about to change numerous business models and organisations profoundly (Büyüközkan and Göçer, 2018). This also applies to the management of supply chains (SCs) where many businesses across a range of industries face various challenges. While there are various definitions of 'Industry 4.0 ' from different 
perspectives (Yang, 2017), according to Hermann, Pentek and Otto (2015), there are four key components of Industry 4.0 identified:

- Cyber-Physical Systems, the fusion of the physical and the virtual world

- Internet of Things, the interaction of 'things' and 'objects' with each other

- Internet of Services, the offer of services via the world wide web

- Smart Factory, assisting people and machines in their jobs

Therefore, in this paper, Industry 4.0 is understood as an umbrella term for a range of concepts and technologies for the organisation of the value chain.

To develop supply chains further, existing 'silos' need to dissolve to build digital supply chain (DSC) networks which are not only highly responsive to changing circumstances but also transparent for all parties involved. This, unavoidably, has an implication on information sharing and decision making across the supply chain. Many companies struggle to achieve the full potential out of their SCs due to various reasons, for example, because of enormous amounts of available data. Due to the diversity of the topics, the paper at hand focuses on the inevitable change within information sharing and decision making across the SC while facing the Digital Transformation and Industry 4.0. A proper strategy is, consequently, necessary. Within this paper, we do not focus on any special technology or approach which is related to the Digital Transformation and Industry 4.0 but their influences on information sharing and decision making and how to cope with them through a proper SC strategy.

Companies need to find a way to cope with these challenges appropriately to ensure their managerial success in the future. Due to the numerous amounts of challenges connected with this topic, difficulties may arise in answering them. This paper picks up these challenges and aims to sharpen possible answers to deal with the 
impact of Industry 4.0 and the digitisation on information sharing and decision making across the SC. Thus, the goal of ultimately formulating an appropriate transformation strategy for SCs is pursued. The authors intend to design a strategy that is general and broadly applicable to a range of industries or companies with different business goals. Against this backdrop, the following research questions reflect these ambitions:

- RQ1: What are the impacts of 'Industry 4.0' and the 'Digital Transformation' on information sharing and decision making across the supply chain?

- RQ2: How can companies manage these impacts on their supply chains and what strategies might be effective to do so?

\section{Methodology}

This paper follows an explorative approach. To address RQ1 and RQ2 we start with an extensive literature review. The literature review follows the guidelines Durach, Kembro and Wieland (2017) suggested. Based on that, interview questions are derived. The questions which we based on the literature review, are formulated as open questions giving the interviewee the possibility to answer individually. The order of the questions is predetermined. After the transcription of all interviews, the analysis is carried out. For the analysis, a qualitative analysis according to Corbin and Strauss (1990) was applied. The unstructured information available suited this procedure well. First, a within-case analysis was carried out in order to understand each companies SCM strategy in order to cope with Industry 4.0 and the digital transformation best. Furthermore, a cross-case analysis was done in order to find patterns. Then, we applied data triangulation through considering insights from the interviews, company documents and company websites. Afterwards, generalizable patterns were identified through cross-case analysis. 


\section{Literature Review}

\section{Supply Chains in the Face of New Technologies}

Over the last decades, SCs became more interrelated and international. Yet, the typical $\mathrm{SC}$ is of long transportation time and low logistics efficiency as Yan (2017) determines.

Major SC challenges are extremely complex nowadays and that simultaneous addressing of these challenges is absolutely necessary (Butner, 2010). To overcome these challenges, SCM needs to become smarter. Therefore, information technology is helpful for the improvement of the SC as it has the potential of integrating various suppliers, customers and processes as well as the ability to enhance the communication in form of data collection and information sharing across the SC (Abdel-Basset, Manogaran and Mohamed, 2018; Zhu et al., 2014).

Against this backdrop, organisations need to adjust their SC to construct a digital supply chain. The objective is to construct a new supply network as Haddud et al. (2017) point out. This kind of new network has the objective 'to create a digital community of partners executing coordinated processes in a more organized and informed way than in the past' (Merlino and Sproge, 2017, p. 309). Therefore, we summarise that to achieve this ambitious goal, we perceive the aid of technology as necessary and Industry 4.0 and the Digital Transformation play a vital role. Consequently, these new developments have impacts on information sharing and decision making across the SC.

\section{Impact on Information Sharing across the Supply Chain}

Information Sharing is commonly cited as the most valuable key for reducing SC connected costs (Khan, Hussain and Saber, 2016). Recently, adoption of new electronic traceability systems by companies takes place to track inventory, sales, purchases as 
well as production for the best possible SCM. Smart SCs are oriented to generate better information which holds various characteristics, ranging from the correct type of information, an improved quality, better timing and speed, ease of access as well as suitable controllability in regard to privacy issues (Wu et al., 2016).

A way to contribute positively to problems concerning information sharing is the IoT. A combination of mobile, cloud and smart systems is a key enabler that can lead to the creation of new types of SCs where physical and digital flows are merged (Barata, Rupino Da Cunha and Stal, 2018). Based on that, companies can transform themselves into real-time firms where the physical and the information flow are integrated $\mathrm{Cu}, \mathrm{Lim}$ and Yang, 2018). Through the mentioned real-time information exchange, enhancement of SC responsiveness can be achieved (Dweekat, Hwang and Park, 2017).

Consequently, this results in cost reductions due to the real-time optimisation, as well as rising the agility of the SC and increased speed of information flow due to resource tracking in real-time as Dweekat, Hwang and Park (2017) picture it.

The collaboration between the parties involved within a SC needs to be managed integrally. Where existing collaboration platforms failed, a cloud-based platform for an improved process of data and information sharing between all stakeholders is a basic requirement for enhanced collaboration across the SC (Gnimpieba et al., 2015; Kohli and Jensen, 2010; Lee and Gao, 2005; Manatsa and McLaren, 2008). Blockchain technology can also help when it comes to creating lasting information transparency within a SC (Azzi, Chamoun and Sokhn, 2019).

Finally, we notice a constant change in regard to information management requirements. A common standard for information transfer as well as communication are inalienable to unlock the full potential globally (Bienhaus and Haddud, 2018). We address this issue through this study. 


\section{Impact on Decision Making across the Supply Chain}

Rezaei, Shirazi and Karimi (2017) point out that there are different levels of decisions within a SC, namely strategic, intermediate and operational decisions that must all be aligned with each other. Generally, within a DSC, we envision all decisions to be holistically and systematically evaluated. Therefore, automation can depict an integrated approach for this plan (Bechtsis et al., 2018). By the usage of information-transmitting devices, a much higher amount of relevant data of various processes can be gathered and used by the decision-maker (Dunke et al., 2018). This can be achieved, for example, through the usage of the RFID technology.

The analysis and interpretation of data and results in real-time support organisations in the process of making faster and better decisions with the overall objective of satisfying their customer requirements (Govindan et al., 2018). The collected data can be analysed and organised to establish a decision support system as well as an awareness of the respective context which can lead to increased coherence and consistency of decisions and, thus, a more efficient decision (Dweekat, Hwang and Park, 2017).

The usage of Big Data within SCM is growing, yet, new tools are required to fully exploit the data and make sense out of it in terms of a more informed decision making (Barata, Rupino Da Cunha and Stal, 2018). Nevertheless, it is generally anticipated that Big Data has a positive impact on SCM as it enables organisations to make more data-oriented, strategic and informed decisions (Addo-Tenkorang and Helo, 2016). Mathematical techniques used in advanced analytics lead to insights that can influence decisions positively (Herden, 2017; Wu et al., 2016).

Although the positive aspects of the digitisation on decision making are obvious, it is anticipated that it will remain important to build personal, human relationships 
across the SC based on mutual trust (Bienhaus and Haddud, 2018). For managing these impacts, a clear strategy in practice is necessary. The paper at hand addresses this issue.

\section{Strategies for the Management of these Impacts}

Industry 4.0 strategies were announced by several European countries which forms the basis for technology roadmaps as well as research agendas (Santos et al., 2017). Examples for this are the 'Cyber-Physical European Roadmap \& Strategy' or the 'European Roadmap for Industrial Process Automation'. However, these existing plans are highly unclear which impedes organisations of all kind to understand these technological concepts and thus make it more difficult to reach their related individual objectives and visions (Santos et al., 2017). Additionally, we perceive these roadmaps to be mostly directed towards manufacturing whereas this paper seeks to research strategies for the SC.

Since no unified and generally accepted definitions of the terms Industry 4.0 and the Digital Transformation exist, logically, organisations have various approaches and strategies in regard to this topic (Bienhaus and Haddud, 2018). For the successful implementation of innovations within a SC, a changed mindset and the communication of a clear strategy in regard to those innovations among all parties involved in the SC is necessary (Sabri, Micheli and Nuur, 2018). Furthermore, according to them, the transformation needs to be considered as a long-term process with cyclical, successive phases consisting of various trials with an organisational nature which is interorganisational and cross-functional.

The literature suggests calculating an Industry 4.0 readiness factor at first (Trstenjak and Cosic, 2017). Afterwards, project phases need to be defined since the transformation takes time and effort according to them. Furthermore, ahead of possible 
investment decisions, Wu et al. (2016) argue that organisations need to think about which SC elements have the potential to generate the largest efficiencies and which technologies are appropriate to exploit them. It is then required to subdivide the project into three phases of implementation. In phase 1, the early phase, the organisation needs to concentrate on local applications, such as RFID and e-sourcing. Then, the intermediate phase 2, deals with isolated applications like smart services or smart factory. The advanced phase focuses on smart applications for the whole SC where sourcing, production as well as retailing are integrated (Wu et al., 2016).

We conclude that the literature review shows that attempts of a strategy for the management of impacts from Industry 4.0 and the Digital Transformation on the SC exist. However, organisations struggle individually to transfer these into practice. In fact, 'there are not any companies that have yet succeeded in truly building DSC, and DSC applications remain limited' (Büyüközkan and Göçer, 2018, p. 164). Although the EU government tries to provide roadmaps for the whole European economy, we can detect a lack of individual strategies broken down to industry types or certain companies to cope with the influences of Industry 4.0 and the Digital Transformation. We try to close this gap through an explorative study with 11 interviews in the following.

\section{Interviews}

Industry 4.0 has its roots in Germany. Germany is one of the most developed industry states with highly developed companies of various backgrounds. The country has one of the highest degree of automation in the world (Eisenkrämer, 2018). In consequence, we decided to interview industry experts solely from Germany in the first step for this paper. 
The semi-structured interviews were conducted in nine different organisations. Overall, findings from 11 interviews are included in the paper at hand (see Figure 1). The case selection was achieved through a sampling approach with two steps: Firstly, a homogeneous origin (German-speaking) was necessary to ensure a comparable exogenous setting. Also, the selected companies needed to be widely open to innovations in regard to their SC. Secondly, we chose companies differentiating in terms of company size (medium, large), owner structure (non-family business, family business) and service scope. Through this process, we tried to make sure to achieve a sample which is heterogenous but to a certain degree homogeneous as well. Thus, we were able to compare and generalize the obtained results.

\begin{tabular}{|c|c|c|}
\hline No. & Industry & No. Of Employees Position Of Expert \\
\hline 1 & Health Care & $>230,000$ Category Manager Strategic Purchasing \\
\hline 2 & Automotive & $>80,000$ Head of Operations Management Material Steering \\
\hline 3 & Automotive & $>80,000$ Head of Inbound Logistics and Plant Logistics \\
\hline 4 & Domestic Appliances & $>60,000$ Head of Organisation and Processes OEM \\
\hline 5 & Tools & $>$ 2,700 Senior Director International Logistics \& Transport \\
\hline 6 & Domestic Appliances & $>60,000$ Commercial Director \\
\hline 7 & Imaging & $>$ 25,000 Project Manager Inbound Supply Chain \\
\hline 8 & Construction Material & $>75,000$ Head of Operations \\
\hline 9 & Logistics Service Provider & $>$ 1,600 Sales Manager - Expert for holistic Logistics Solutions \\
\hline 10 & Semiconductors & $>$ 35,000 Supply Chain Digitalisation Expert \\
\hline 11 & Online Shopping & $>550,000$ Robotics Manager \\
\hline
\end{tabular}

Figure 1: Case overview

\section{Analysis}

Research revealed that in practice, most necessary information is already available.

However, there are barely appropriate possibilities to analyse this data to make use out of it. Much information exists in different formats which is one of the major problems as it impedes the usage and processing. Furthermore, a misjudgement of many companies in terms of their data may exist. Either companies collect the wrong data, they collect data of low quality or data which is not representative of any kind of analysis. The interviewees attested that through improved information sharing, various 
processes can be automated based on a highly accurate forecast. However, a central aspect is to improve the quality of information. Only based on reliable information, these improved processes are possible. A further central aspect is the centralisation of information and the sharing of them. Where currently a lot of different standalone solutions are in use, the future needs to be centralised, to improve and slim down the information sharing process.

As stated from all interviewees without exception, the overall aim is to achieve a whole SC integration in the future through Industry 4.0 related technologies which means a total information exchange starting from sub-sub-suppliers up to the endcustomer. This may be achieved through a centralised platform where every party involved is connected. Otherwise, it is extremely important to establish standards for this new kind of information exchange, whereas currently, many companies are developing their systems based on their enterprise resource planning systems. Therefore, the solving of the problems in terms of the various interface is a central challenge.

Additionally, interviewees mentioned that a lot of information is lost at the company border as currently companies are not ready and do not want to exchange huge amounts of far-reaching information as they may fear disadvantages from that ("There is a lot of potentials to exploit, but it usually is not exhausted", Interview No. 10). Currently, companies may fear that they would fall behind their competitors by sharing too much information with their partners. Nevertheless, problems are anticipated with getting very small partners connected to a centralised platform as it may be barely achievable for small businesses to invest in their IT-systems ("There are a lot of suppliers who work analogue up to now", Interview No. 5). Regarding data protection, this development was perceived as uncritically from the interviewees as the European 
Union legally regulates these processes, e.g. through the 'General Data Protection Regulation (GDPR) (EU) 2016/679'. Generally, we noted from the interviews that the Industry 4.0 related technologies all communicate with each other more and more and that through smart, real-time based information processing, more holistic information is available for a smarter SCM.

Our research shows that decision-making processes across the SC are greatly connected with the information sharing process as most decisions are based on the available information at that point in time. For example, through Industry 4.0 influenced and improved information sharing, companies may be able to plan more accurately, e.g. in terms of arriving trucks. This means that, through new technologies, companies are enabled to track the movement of trucks more easily and use this information to predict arrival times very accurately. However, as also mentioned before, this is affected profoundly by information and data quality. Several quality gates for information sharing as a basis for the decision-making process were suggested. Furthermore, Neural Networks may be more efficient and effective than humans in generating an optimised forecast based on more accurate data. These forecasts can then influence business decision making profoundly and can lead to higher productivity and efficiency. On the contrary, interviewees mentioned that due to more and more available information based on Industry 4.0 related technologies, an increasing amount of internal coordination meetings is necessary. This means that a well internal collaboration across many different departments is one of the key factors of success in the future in terms of decision making. Predominantly, most interviewees anticipated a vast amount of recommendations for actions through IT-systems. Due to the real-time information processing, IT-systems were anticipated to generate better decisions than, for example, a human SC planner. The human being may have a lot of experience, nevertheless, the 
amount of information which a human being can process is limited and therefore, ITsystems may make valuable recommendations ("Humans are so far often superior to ITsystems due to intuition", Interview No. 4). However, full automation was not expected soon, since there is some scepticism about fully automated decisions of IT-systems. This means that currently, many companies tend to implement systems for recommendations. However, the last confirmation and decision are made by a human being. Machines are also expected to be capable to make complicated decisions that are based on Deep Learning and Machine Learning algorithms. Currently, the strategy is more to bring the best out of the two worlds, the human and the artificial, together, in order to achieve the best possible decisions. It is still necessary as well as wanted in some cases, that humans monitor decisions and recommendations for action from computer systems ("There are a lot of individual orders which need to be processed through a human being", Interview No. 7). Consequently, the human being can decide either the same or different from the system. However, all decisions made or recommended artificially must be in alignment with the company's individual compliance rules, which is certainly very important. Nevertheless, through recommendations from IT-systems, it is one of the objectives to reduce the individual room for manoeuvre of the individual employee. This can help to improve the stability of various processes. Nevertheless, a certain scepticism in regard to the data should always be maintained.

In regard to Transformation Strategies for the SC, each interviewee's company had a different strategy to cope with the indispensable impacts of Industry 4.0 and the Digital Transformation on their business. Some companies had no central strategy for their digital journey which must also be perceived as a kind of strategy. These organisations have a very rapid living culture where every employee can propose ideas 
and contribute to this development. This means that a kind of 'Just Do It' culture shapes their strategy with extremely fast adjustments and implementations of new processes and ideas. Through the faster and faster environment, companies need to adjust more and more rapidly which may make a long-term strategy obsolete as nobody can anticipate exactly what will happen in ten or more years. In comparison, other companies had also no clear strategy communicated in terms of Industry 4.0 and the Digital Transformation. In this case, however, this seemed to contribute to certain insecurity among the employees. A clear objective was not visible.

Yet, companies predominantly prepared for the future with a classical multi-year strategy. First of all, an analysis of the current state is done and based on that, a vision for the future is generated which shows the imagination in terms of digital impacts. Then, a concrete schedule is derived for the implementation. As part of such a strategy, also strategic partnerships with long-term business partners are necessary, e.g. for the development of new digital products and services. Such an overall vision for the entire company can then be broken down into concrete steps to achieve the vision. These steps are then fulfilled by extensive projects leading to the ideal state of the future. Otherwise, some companies are planning based on operative key performance indicators such as, for example, the turnover of the corporate turnover. For instance, a certain increase of the turnover is planned for the next years and to achieve this goal, these companies are looking at digital opportunities such as Industry 4.0 related technologies. This means that Industry 4.0 and the Digital Transformation are more or less only means of the purpose and less a specific area where their companies want to excel to achieve the overall goal of customer satisfaction. 


\section{Discussion}

Our research shows that there is still a lack of a unified definition of terms such as 'Industry 4.0' and the 'Digital Transformation' which was described in academia before, e.g. from Hofmann and Rüsch (2017). Through this uncertainty regarding the interpretations of the key terms, we perceived that many practitioners from various kinds of industries and hierarchical levels were insecure about their level of understanding. Although the understanding of the key terms and the accompanied trends of digitisation across the SC vary greatly, companies have to find out which technologies are useful for them and generate the largest benefits for their business case. Therefore, the return on investment is a central performance indicator to evaluate new technological implementation ideas. The reason behind this is the high investment burden which especially small and medium-sized companies are confronted with to stay competitive. However, even small businesses need to invest in Industry 4.0 related technologies to stay up-to-date to avoid to be put out of business.

Furthermore, individual solutions hinder the information sharing process across the SC as the communication of information systems is usually based on standard interfaces which are not given by individual tools. Many information is lost at the company border as various interviewees confirm. Therefore, it is critical that the information sharing processes across the SC are not optimised so far, but it is mostly focused only on the internal information exchange. Hence, we can conclude that the fully central SC integration as one of the main advantages based on the utilisation of Industry 4.0 related technologies seems to be still at distance for many companies. To achieve this and all of its advantages must be one of the key goals for every SCM. We believe that the reason why companies are not bold enough to share appropriate information with their partners can be imagined as anxiety to fall behind their 
competitors or to be exploited by their partners. Strategic partnerships with partners who have known each other for a long time may help to set off this process in the right direction. High-security standards regarding the shared information are expected to help to achieve a full SC integration based on Industry 4.0 technologies.

Although the information sharing processes within many SCs are not optimised as our research shows, more and more recommendations for action are based on SC related information and data. Companies follow the general trend of utilising data as a basis for SC related decisions. Simple decisions are often automated. However, the findings revealed less automation concerning decision making than expected. Many companies seem to be sceptical about fully automated complex decisions. Yet, generally, this more fact-based method may lead to cost savings and better decisions in general. According to our findings, most companies use a phase-based strategy which is common across industries. Nevertheless, there is no specific digital SCM related strategy communicated in various enterprises.

One of our objectives with the paper at hand is the suggestion of a transformation strategy for the SC towards a DSC or a so-called Supply Chain 4.0. As Sabri, Micheli and Nuur (2018) pointed out, the transformation needs to be considered as a long-term process with cyclical, successive phases consisting of various trials with an organisational nature which is inter-organisational and cross-functional. In conjunction with the various transformation strategies found out empirically, we developed the following generalized strategy as a suggestion which needs to be validated in the future (see Figure 2). 


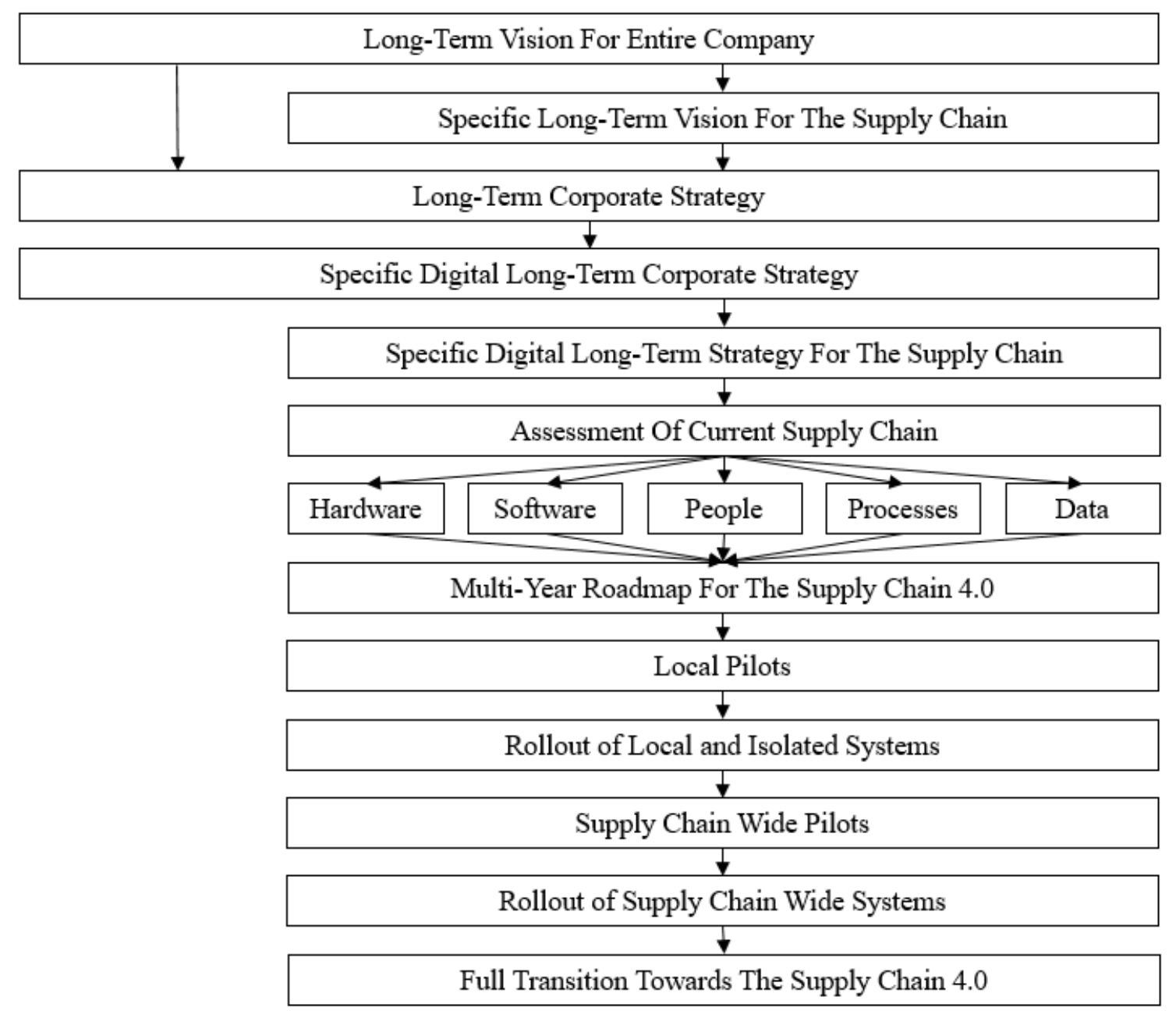

Figure 2: SC Transformation Strategy

We suggest that the first step is to establish a long-term vision for the entire company. This vision needs to visualise the long-term goals of the company and how the management envisions the future optimal state. Then, in alignment with the overall vision, a specific SC related vision is necessary to specify how the SC of the future should look like. The suggested timeframe for these visions is between 15 to 30 years. Based on the visions, a corporate strategy needs to be created which shows what steps are necessary to reach the future optimal state envisioned. Again, in alignment with this strategy for the entire company, a dedicated digital corporate strategy needs to be formed. 'Siloed' strategies must be avoided under all circumstances. The digital strategy can then be divided into a specific SC related digital long-term strategy that 
incorporates the plans for the future state of the SC. We suggest communicating the specific strategy towards the employees to integrate their ideas as well. Then, the actual strategic work on the transformation of the SC may start. First of all, the current state of the SC needs to be assessed. The appointment of a dedicated SC transition project team is recommended. We suggest doing this assessment in various dimensions to consider the various transformation requirements. The suggested dimensions are 'Hardware', 'Software', 'People', 'Processes' and 'Data'. Having considered these aspects, a final multi-year roadmap for the transformation of the SC must be generated. This roadmap needs to incorporate concrete steps to achieve the full transformation of the SC towards the optimal state in the future. We suggest prioritising these steps according to the overall transformation strategy. Ahead of the pilots and the connected investment decisions, companies need to figure out which technologies promise the largest benefit based on their circumstances. Successful pilots may then be implemented and rolled out locally. The attempt to get inspired by a start-up culture can be useful here to guarantee rapid implementations. This means that isolated systems should then be in practice which may then, in the following step, be expanded throughout the whole SC. Therefore, SC wide pilots must then be set up. Strategic partnerships with long-term business partners are necessary at this stage of the transformation. Consequently, successful SC wide pilots may then be implemented alongside the entire SC. This full integration throughout the entire SC then leads to the full transformation towards a Supply Chain 4.0. Last but not least, it must be noted that the entire vision and strategy, not only for the SC but also for the entire company, needs to be revisited at regular intervals. Through this holistic approach, problems with regard to the coordination of various Industry 4.0-related initiatives within a company should be overcome. Our strategy may be enhanced through calling in other models of Industry 4.0 
implementation such as Telukdarie et al. (2018) suggested even though a focus on manufacturing processes may exist.

\section{Conclusion}

The contributions and implications for the practice of this paper are threefold. First, the impact of the Digital Transformation and Industry 4.0 on information sharing was investigated in detail. New possibilities originate for many companies. Issues addressed e.g. are predictive maintenance through smart products, predictive analytics for SC risk management, smart labour planning through improved forecasts based on new and better-utilised information or customer individual products based on configurators and 3D Printing. Our research showed that Industry 4.0 and the Digital Transformation have the potential to achieve a full digital SC integration in terms of higher transparency, e.g. through centralised platforms. However, up to now, companies hesitate to share farreaching information with their business partners which mean that a lot of information is lost at the company border, although the information exchange would be beneficial for all parties involved. Hence, companies need to open up to achieve the full advantages. A central problem of the digitisation of processes across the SC in terms of the information exchange between business partners is the lack of standards for the information sharing process from a technological point of view. Currently, many companies implemented various IT-tools and platforms, either from external specialised software manufacturers or based on own developments. However, there are no standards across industries for the information sharing interface between companies' internal information systems. Therefore, for practitioners, one implication of our research may be that is of crucial importance, to establish standards for the information exchange which enables even small and midsized companies to participate to create 
overall transparency for the SC.

Secondly, the impact of the Digital Transformation and Industry 4.0 on decision making was examined with the following key findings: The decision-making process across the SC is more and more connected to information sharing throughout the SC. This means that an increasing amount of decisions is made based on available data and are, therefore, data-driven. Consequently, the information quality, quantity, and accuracy are of pivotal importance. Fewer mistakes and improved planning security are anticipated. More and more recommendations for action through Industry 4.0 related technologies are perceived and expected across various industries. An increasing amount of simple decisions is already automated which is due to rule-based approaches. Through these technology-supported decisions and also through new possibilities in terms of forecasting, it is expected that decision-making across the SC is improved and leads ultimately to higher productivity and efficiency. However, most companies see the full automation of complicated SC related decisions influenced by Industry 4.0 related technologies critical. Up to now, in most cases, a human being is still necessary and also wanted on purpose for the last confirmation or overdriving automated recommendations and actual decisions of information systems. From a practitioner's perspective, it should be judged in detail which decisions can be automated through Industry 4.0 related technologies. This has ultimately consequence for personnel planning processes.

Our research identified several key transformation strategies that differ greatly: Firstly, we discovered classical multiyear strategies, sometimes backed-up with a longterm vision, is predominantly used in the industry. A special-purpose strategy in terms of digital objectives may be derived from an overall strategy for the entire company. These strategies are mostly divided into several project phases, starting with a classical situation analysis and ending with an envisioned, optimal future state. Based on the 
findings we laid out an own strategy proposal. Secondly, another approach is the rapid implementation of necessary improvements in terms of new Industry 4.0 related technologies. This means that some companies do not make use of specific DSC related strategies but rather attempt to stay at the current state of the art to remain competitive in the future. This mainly requires extremely fast decision making in terms of the implementation of new technological solutions depending on a combination of bottomup and top-down planned projects. The third type of strategy which was found out is to achieve the general objectives in terms of certain key performance indicators. This approach also does not provide a specific Industry 4.0 related strategy for the SC but utilises Industry 4.0 related technologies as a means for the purpose to reach the overall goals of the company. The implication of the paper for the practitioners can be to think in detail about a DSC specific strategy in order to cope with the challenges arising through the Digital Transformation and Industry 4.0 in terms of information sharing and decision making across the SC. We consider this issue of pivotal importance for the lasting success of an SC of the future.

However, the paper at hand has several limitations: The reviewed documents within the literature review are based on a certain, subjective selection on academic journals. Through the inclusion of additional literature, the findings of the review can be enriched in the future. A different approach to collating the relevant studies may be utilised. All semi-structured interviews were carried out in Germany. Therefore, limitations exist in regard to the geographical location. Other experts need to be interviewed for validation purposes, e.g. from the US, Singapore or other highly developed countries. Validation of the proposed strategy needs to be carried out in future research. Furthermore, the qualitative research approach comes with limitations 
itself. Only 11 interviews were done which is a limitation to consider. A large quantitative survey would substantiate the findings.

We conclude that the impact of Industry 4.0 and the Digital Transformation on SCM is far from being fully researched as these concepts are mainly focused on manufacturing. Thus, companies need to prepare for the arising challenges. Therefore, a range of areas still exists which require attention for future research. The research can serve as a starting point and maybe laid out on a large-scale basis and put into a wider perspective. Moreover, practitioners from different industrial backgrounds might state different opinions that need to be examined by future research. Little was said about the impact of Industry 4.0 and the Digital Transformation on legal aspects throughout the SC. Financial aspects were also mostly not taken into consideration. Hence, future research must examine these potential areas of conflict.

Acknowledgements

We would like to thank all anonymous interviewees for their time and input.

Abdel-Basset, M., Manogaran, G. and Mohamed, M. (2018) 'Internet of Things (IoT) and its impact on supply chain: A framework for building smart, secure and efficient systems', Future Generation Computer Systems, 86, pp. 614-628. doi: 10.1016/j.future.2018.04.051

Addo-Tenkorang, R. and Helo, P.T. (2016) 'Big data applications in operations/supplychain management: A literature review', Computers \& Industrial Engineering, 101, pp. 528-543. doi: 10.1016/j.cie.2016.09.023

Azzi, R., Chamoun, R.K. and Sokhn, M. (2019) 'The power of a blockchain-based supply chain', Computers \& Industrial Engineering, 135, pp. 582-592.

doi: 10.1016/j.cie.2019.06.042 
Barata, J., Rupino Da Cunha, P. and Stal, J. (2018) 'Mobile supply chain management in the Industry 4.0 era: An annotated bibliography and guide for future research', Journal of Enterprise Information Management, 31(1), pp. 173-192.

doi: 10.1108/JEIM-09-2016-0156

Bechtsis, D. et al. (2018) 'Intelligent Autonomous Vehicles in digital supply chains: A framework for integrating innovations towards sustainable value networks', Journal of Cleaner Production, 181, pp. 60-71.

doi: 10.1016/j.jclepro.2018.01.173

Bienhaus, F. and Haddud, A. (2018) 'Procurement 4.0: factors influencing the digitisation of procurement and supply chains', Business Process Management Journal, 24(4), pp. 965-984. doi: 10.1108/BPMJ-06-2017-0139

Butner, K. (2010) 'The smarter supply chain of the future', Strategy \& Leadership, 38(1), pp. 22-31. doi: 10.1108/10878571011009859

Büyüközkan, G. and Göçer, F. (2018) 'Digital Supply Chain: Literature review and a proposed framework for future research', Computers in Industry, 97, pp. 157-177. doi: 10.1016/j.compind.2018.02.010

Corbin, J.M. and Strauss, A. (1990) 'Grounded theory research: Procedures, canons, and evaluative criteria', Qualitative Sociology, 13(1), pp. 3-21. doi: 10.1007/BF00988593

Dunke, F. et al. (2018) 'Time traps in supply chains: Is optimal still good enough?' European Journal of Operational Research, 264(3), pp. 813-829. doi: 10.1016/j.ejor.2016.07.016

Durach, C.F., Kembro, J. and Wieland, A. (2017) 'A New Paradigm for Systematic Literature Reviews in Supply Chain Management', Journal of Supply Chain Management, 53(4), pp. 67-85. doi: 10.1111/jscm.12145 
Dweekat, A.J., Hwang, G. and Park, J. (2017) ‘A supply chain performance measurement approach using the internet of things', Industrial Management \& Data Systems, 117(2), pp. 267-286. doi: 10.1108/IMDS-03-2016-0096

Eisenkrämer, S. (2018) Deutsche Industrie liegt bei Automatisierung weit vorne. Available at: https://www.springerprofessional.de/automatisierung/ industrieroboter/deutsche-industrie-liegt-bei-automatisierung-weit-vorne/ 15446374 (Accessed: 18 Februar 2019).

Gnimpieba, Z.D.R. et al. (2015) 'Using Internet of Things Technologies for a Collaborative Supply Chain: Application to Tracking of Pallets and Containers', Procedia Computer Science, 56, pp. 550-557. doi: 10.1016/j.procs.2015.07.251

Govindan, K. et al. (2018) 'Big data analytics and application for logistics and supply chain management', Transportation Research Part E: Logistics and Transportation Review, 114, pp. 343-349. doi: 10.1016/j.tre.2018.03.011

Haddud, A. et al. (2017) 'Examining potential benefits and challenges associated with the Internet of Things integration in supply chains', Journal of Manufacturing Technology Management, 28(8), pp. 1055-1085. doi: 10.1108/JMTM-05-20170094

Herden, T.T. (2017) 'Similarity-matching in decision-making processes of Supply Chain Analytics: a systematic literature review', Supply Chain Forum: An International Journal, 18(1), pp. 13-20. doi: 10.1080/16258312.2017.1278670

Hermann, M., Pentek, T. and Otto, B. (2015) Design Principles for Industrie 4.0 Scenarios: A Literature Review, 27 Juni. Available at: http:// www.thiagobranquinho.com/wp-content/uploads/2016/11/Design-Principles-forIndustrie-4_0-Scenarios.pdf. 
Khan, M., Hussain, M. and Saber, H.M. (2016) 'Information sharing in a sustainable supply chain', International Journal of Production Economics, 181, pp. 208-214. doi: 10.1016/j.ijpe.2016.04.010

Kohli, A.S. and Jensen, J.B. (2010) 'Assessing Effectiveness of Supply Chain Collaboration: An Empirical Study', Supply Chain Forum: An International Journal, 11(2), pp. 2-16. doi: 10.1080/16258312.2010.11517228

Lee, J.D. and Gao, J. (2005) 'Trust, Information Technology, and Cooperation in Supply Chains', Supply Chain Forum: An International Journal, 6(2), pp. 82-89. doi: $10.1080 / 16258312.2005 .11517150$

Manatsa, P.R. and McLaren, T.S. (2008) 'Information Sharing in a Supply Chain: Using Agency Theory to Guide the Design of Incentives', Supply Chain Forum: An International Journal, 9(1), pp. 18-26. doi: 10.1080/16258312.2008.11517187

Merlino, M. and Sproge, I. (2017) 'The Augmented Supply Chain', Procedia Engineering, 178, pp. 308-318. doi: 10.1016/j.proeng.2017.01.053

Rezaei, M., Shirazi, M.A. and Karimi, B. (2017) 'IoT-based framework for performance measurement: A real-time supply chain decision alignment', Industrial Management \& Data Systems, 117(4), pp. 688-712. doi: 10.1108/IMDS-08-20160331

Sabri, Y., Micheli, G.J.L. and Nuur, C. (2018) 'Exploring the impact of innovation implementation on supply chain configuration', Journal of Engineering and Technology Management. doi: 10.1016/j.jengtecman.2018.06.001

Santos, C. et al. (2017) 'Towards Industry 4.0: an overview of European strategic roadmaps', Procedia Manufacturing, 13, pp. 972-979. doi: 10.1016/j.promfg.2017.09.093 
Telukdarie, A. et al. (2018) 'Industry 4.0 Implementation for Multinationals', Process Safety and Environmental Protection, 118, pp. 316-329. doi: 10.1016/j.psep.2018.06.030

Trstenjak, M. and Cosic, P. (2017) 'Process Planning in Industry 4.0 Environment', Procedia Manufacturing, 11, pp. 1744-1750. doi: 10.1016/j.promfg.2017.07.303

Tu, M., Lim, M.K. and Yang, M.-F. (2018) 'IoT-based production logistics and supply chain system - Part 1: Modeling IoT-based manufacturing supply chain', Industrial Management \& Data Systems, 118(1), pp. 65-95. doi: 10.1108/IMDS11-2016-0503

Wu, L. et al. (2016) 'Smart supply chain management: a review and implications for future research', The International Journal of Logistics Management, 27(2), pp. 395-417. Available at: https://doi.org/10.1108/IJLM-02-2014-0035.

Yang, L. (2017) 'Industry 4.0: A survey on technologies, applications and open research issues', Journal of Industrial Information Integration (6), pp. 1-10.

Zhu, D.S. et al. (2014) 'Will You Share? A Study of Motives and Consequences of Supply Chain Partners' Information Integration', Supply Chain Forum: An International Journal, 15(2), pp. 4-18. doi: 10.1080/16258312.2014.11517338 
Raphael Preindl is a PhD candidate at the University of St.Gallen, Switzerland. He received a M.Sc. in Business Analytics from the University of Manchester, UK, a B.A. in Business Administration from the Baden-Wuerttemberg Cooperative State University, Germany, and a B.A. in Logistics and Supply Chain Management from the University of South Wales, UK.

Prof. Konstantinos Nikolopoulos Dr.Eng. ITP P2P, holds the Chair in Business Analytics in Bangor Business School and is the Director of the forLAB forecasting laboratory (www.forLAB.eu); he also has several years of experience in the UK and Greece as Consultant and Software Engineer in projects with Siemens Healthcare Diagnostics, DoH, RevOil and the Treasury and Home Office (in Greece).

Mrs Konstantia Litsiou , BA (Athens), PGCE (Athens), MA (Lancaster) is a Senior Lecturer in Retail Management in Manchester Metropolitan University Business School. Konstantia is also pursuing a PhD in "Forecasting with Judgmental Methods the success of Megaprojects" in Salford Business School. Konstantia has a background in arts and has worked in big scale art projects and events in Greece for many years. Her work has appeared in international journals and conferences. 